\title{
Development of a New Approach for the Assessment of Complex Blood Flow Patterns in the Left Atrium From 4D Flow MRI
}

\author{
Enrico Soldati ${ }^{1}$, Thomas Dietenbeck ${ }^{2}$, Alban Redheuil ${ }^{3}$, Alessandro Masci ${ }^{1}$, Sophia Houriez ${ }^{2}$, \\ Gombaud Saintonge $^{2}$, Nadjia Kachenoura ${ }^{2}$, Cristiana Corsi ${ }^{1}$ \\ ${ }^{1}$ DEI, University of Bologna, Campus of Cesena, Bologna, Italy \\ ${ }^{2} \mathrm{LiB}$, University of Pierre et Marie Curie, Sorbonne University, Paris, France \\ ${ }^{3}$ Department of Cardiology, Hôpital de la Pitié et Salpetriére, Paris, France
}

\begin{abstract}
Mitral Valve Regurgitation affects 2-3\% of the general population. The morphological and functional remodeling of the left atrium caused by reflowing blood into the LA chamber favours blood stasis and stroke risk. In this context, several clinical studies suggest that stoke risk stratification could be improved by using anatomical, morphological and hemodynamic information on the left atrium. The aim of this study was therefore to develop a new approach for the assessment of complex blood flow patterns in the left atrium using $4 D$ Flow MRI images. This information might help in quantifying the hemodynamic implications of the mitral valve regurgitation on sick patients compared to healthy volunteers. In this paper, we present the developed model as well as its application to patients as a preliminary step forward to an optimized stroke risk stratification and therapy delivery.
\end{abstract}

\section{Introduction}

Mitral valve regurgitation (MVR) is a common disorder affecting $2-3 \%$ of the general population. It is defined as a systolic retrograde flow from the left ventricle (LV) into the left atrium (LA). It is characterized by typical fibromyxomatous changes in the mitral leaflet tissue with superior displacement of one or both leaflets into the left atrium. Despite being known for more than a century, this disease may remain silent until the grade of reflowing blood becomes moderate or severe [1]. The morphological and functional remodelling of the LA caused by this disease could favour blood stagnation and consequently stroke risk. Several clinical studies suggest that stroke risk stratification may be improved by using hemodynamic information of the LA [2].

Cardiovascular magnetic resonance imaging (MRI) offers an excellent anatomical coverage and allows an accurate estimation of atrial geometry as well as blood flow derived indices. Recently, a dynamic MRI sequence with velocity encoding in the three-dimensional space and with a full three-dimensional anatomical coverage (4D flow MRI), opening a new area of research in terms of cardiovascular complex flow visualization and quantification [3]. Flow quantification with 4D flow MRI has several advantages since it allows 1) the investigation of the internal consistency of the data by employing the principle of mass conservation; 2) the retrospective placement of analysis planes at any location within the acquisition volume and offers the ability to retrospectively calculate blood flow through any plane of interest across the 3D volume; 3) the visualization of multi-directional flow features and alterations in presence of cardiovascular disease [4].

4D flow MRI has been used in several studies for the estimation of aortic and ventricular volumes and the evaluation of blood flow features [5]. Despite the relevance of the problem, there are no studies using 4D flow MRI data analysis on patients affected by MVR. Therefore, the aim of this study is to develop a new approach for the assessment of complex blood flow patterns in the LA using 4D flow MRI which could quantify the hemodynamic implications of the MVR on patients compared to healthy volunteers. We introduced several original contributions: patient specific LA anatomical model of two healthy and two MVR patients were extracted by a semi-automatic image segmentation algorithm from 4D flow MR images and haemodynamic parameters were directly extrapolated from the data.

\section{Material and Methods}

\subsection{Patient Data}

Clinical data were provided by Hôpital de la Pitié et Salpetriére, Laboratoire d'Imagerie Biomédicale, Université Pierre et Marie Curie, Sorbonne Universités, Paris. In particular, in our preliminary study we selected 2 patients affected by MVR and 2 healthy volunteers. 
Cardiac 4D flow MRI images were acquired using a Siemens 1.5T MRI scanner applying T1-weighted sequence. Twenty volumes (pixel size $1.77 \mathrm{~mm}$, slice thickness $2.4 \mathrm{~mm}$ ) spanning one cardiac cycle from the end of (ventricular) diastole were reconstructed by retrospective ECG and respiratory gating.

\subsection{LA Segmentation}

In order to process the data, we developed a specific workflow described in Figure 1.

\begin{tabular}{|c|c|c|}
\hline $\begin{array}{l}\text { 4D flow } \\
\text { MRI }\end{array}$ & $\begin{array}{c}\text { LA } \\
\text { segmentation }\end{array}$ & $\begin{array}{c}\text { Velocity field extrapolation } \\
\text { and features analysis }\end{array}$ \\
\hline
\end{tabular}

Figure 1. Project workflow.

4D flow MRI were processed to extract the 3D LA anatomical model and to extrapolate the velocity field and to compute the kinetic energy (KE) within the LA.

To optimize the performance of the segmentation step, we first computed a $3 \mathrm{D}$ mask to roughly exclude the regions outside the volume of interest. For each time frame we calculated an angiogram by multiplying the volume of the modulus by the square root of the sum of the squared velocities in the three-dimensional space directions. In addition, we employed morphological operators in order to open a large region around the LA and exclude from the analysis the aorta and the big vessels. The first and last slice of the volume in which the atrium was visible inside the acquired stack were automatically selected.

The model proposed by Chan and Vese was applied to detecte the LA chamber. If we consider an image $I$ and $C$ a closed regular curve, the Chan-Vese segmentation is based on the minimization of the following functional:

$$
\begin{aligned}
& F_{1}(C)+F_{2}(C)= \int_{\text {inside }(C)}\left|I(x, y)-c_{1}\right|^{2} d x d y \\
&+\int_{\text {outside }(C)}\left|I(x, y)-c_{2}\right|^{2} d x d y \\
& \text { inf }_{c}\left\{F_{1}(C)+F_{2}(C)\right\} \approx 0 \approx F_{1}\left(C_{0}\right)+F_{2}\left(C_{0}\right)
\end{aligned}
$$

where $I(x, y)$ is the grey level intensity image and the constants $c_{1}$ and $c_{2}$ depend on the curve $\mathrm{C}$ and they are, respectively, the mean of $\mathrm{I}$ inside and outside of $\mathrm{C}$. Therefore, the function $\left(F_{1}(C)+F_{2}(C)\right)$ is minimized when $C=C_{0}[6]$.

At the end of the segmentation step, results were optimized by applying opening morphological operators. Finally, we reconstructed the 3D LA anatomical model by stacking the $2 \mathrm{D}$ segmentations. The LA volume was then derived considering the slice thickness and the pixel space in $\mathrm{x}$ and $\mathrm{y}$ directions.

Figure 2 illustrates the final LA anatomical model, where the information of the main anatomy regions is reported.

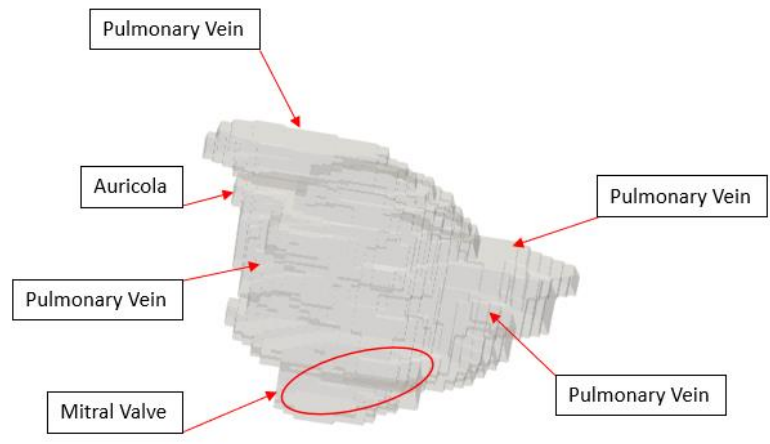

Figure 2. 3D segmented volume of the LA with information of the four pulmonary veins, mitral valve and appendage.

\subsection{Velocity Field}

Velocities inside the LA chamber were directly obtained by the 4D flow MRI. This information was saved as four 4D data: the modulus and the velocity field in $\mathrm{x}, \mathrm{y}$ and $\mathrm{z}$ direction. Regarding the 4D data of velocities, these are structured as $3 \mathrm{D}$ volumes with the intensity of the velocity for each pixel throughout the cardiac cycle.

The data from the segmentation previously obtained were saved as a 4D binary mask. Therefore, velocities were evaluated exclusively inside the region of interest (ROI) by simply multiplying the three 4D volume, containing the intensity of $x, y$ and $z$ velocity by the LA 4D binary mask derived from the segmentation step. Then, the modulus of the velocity inside the LA chamber was computed.

\subsection{Kinetic Energy}

Blood flow patterns in the LA are closely connected to the shape and motion of the thin myocardium layer, including pulmonary veins, and may therefore be a sensitive marker of cardiac function and dysfunction. KE is a quantitative measure of blood flow and many studies have shown that it provides information on the contractile function within the LA and increases the moderate heart failure contour compared to healthy controls. In addition, studies performed on the LV flow component and on patient presenting a range of normal to mild $\mathrm{LV}$ remodelling and normal to mildly depressed LV systolic function, have shown that in remodelled, dysfunctional ventricles, a shift occurred whereby the volume and the 
$\mathrm{KE}$ of the direct flow diminish, while that of the nonejection components increases.

$\mathrm{KE}$, following previously published methods, was simply calculated by the formula:

$$
K E=\frac{1}{2} \operatorname{mass}_{\text {voxel }} V^{2}
$$

where the mass voxel is calculated by multiplying blood density $\left(1.06 \mathrm{~g} / \mathrm{cm}^{\wedge} 2\right)$ by the voxel size $(1.77 \times 1.77 \times 2.40$ $\mathrm{mm}^{3}$ ), and $\mathrm{V}$ is the modulus of the velocity $[7,8,9]$.

Measurements of intracardiac KE provide distinct pathophysiological insight relevant to both diagnosis and therapy of heart failure. Moreover, it empowers the detection of ventricular and atrial dysfunction in clinically compensated patients and is strictly linked to LA contractile function.

\section{Results and Discussion}

In Figure 3, an example of difference between the volumes of sick patient and healthy volunteers is shown:

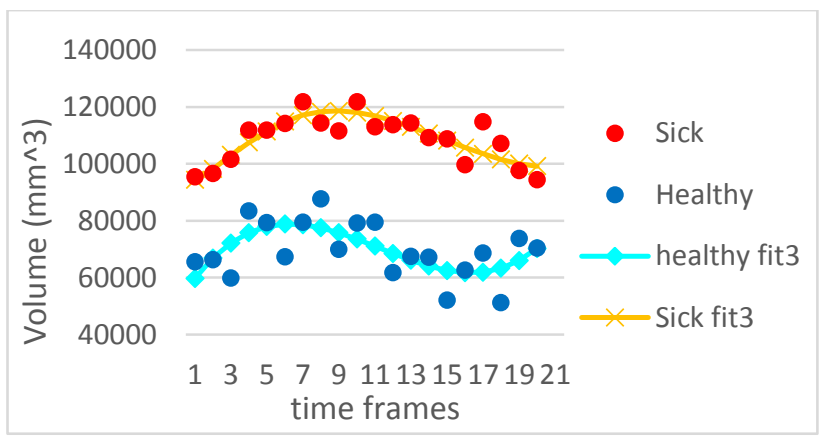

Figure 3. LA volumes for a healthy subject and a sick patient through the cardiac cycle.

According to previous studies, the analysis of the LA volumes showed that those of healthy volunteers were different with respect to sick patients [10]. Bigger volumes in MVR patients were a consequence and an indicator of the disease. In particular, in patients with MVR, this volume enlargement was caused by a blood overload within the LA chamber during the ventricular systole. The overload caused an increase of the pressure at the wall, employing a stretching and a dilatation to decrease the intra-chamber pressure.

From literature, we know that velocities inside the LA in patients affected by MVR were lower than blood velocities in healthy volunteers. Indeed, by evaluating the mean and median velocities, we confirmed the observation reported in these studies [11]. In Figure 4 we present two atria (the healthy on the left and the sick on the right) in a particular time frame corresponding to the $\mathrm{S}$ wave of the atrial cycle, where the mitral valve should be closed because this is the time instant characterized by the LV systole. We noticed that, during the $\mathrm{S}$ wave, in healthy volunteers higher velocities were localized near the pulmonary veins and more in general in the ceiling of the LA, while for MVR patients high velocities were also present in proximity of the MV caused by the regurgitant flow. However, during the time frames where the MV was open, high velocities in correspondence of MV and pulmonary veins were detected in both healthy and sick patients.
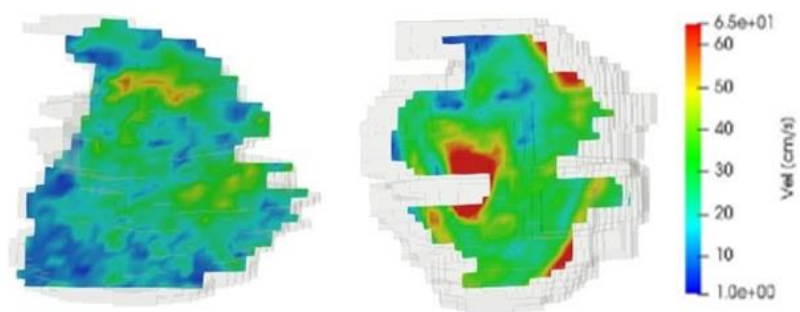

Figure 4. Analysis of the velocity field in the LA in a healthy volunteer (on the left) and a MVR patient (on the right).

The regions with greater $\mathrm{KE}$ are those where the velocities were higher. Therefore, as expected, the higher $\mathrm{KE}$ was found to be close to pulmonary veins and the mitral valve.

In Figure 5, we present the $\mathrm{KE}$ of one healthy volunteer (first row) and a MVR patient (second row) during three main time instants considered. These correspond to $\mathrm{S}$ wave, where the MV is closed, E wave, where the blood flows directly from the pulmonary veins through the MV and the A wave which represents the phase where the atrium contracts, pushing blood actively inside the LV.

For healthy volunteers, the regions with high KE were well defined and closer to pulmonary veins in correspondence of the $\mathrm{S}$ wave. Moreover, we did not find specific KE high values in the center of the chamber and at the MV.

During the $\mathrm{E}$ wave the $\mathrm{KE}$ is higher in proximity of the mitral valve, where the blood entered directly into the LV.

Looking at the A wave, we observed that there were not particular regions with high $\mathrm{KE}$, which was low and uniformly distributed in the chamber. Moreover, in the MVR patients we found regions with high $\mathrm{KE}$ on the mitral valve in correspondence of the $S$ wave, confirming that backflows occur in the LA during ventricular systole, which were not present in the healthy group. Furthermore, in correspondence of the $\mathrm{E}$ wave a more disorganized blood flow lead to lower KE; this means that we were not able to discriminate high values on the mitral valve in sick patients with respect to the healthy volunteers. Moreover, KE in correspondence of A wave 
was very similar for both groups without any particular regions with high values of KE. Because of MVR, the atrium dilates and its walls thicken. Probably due to these effects, the booster pump function of the LA is preserved.

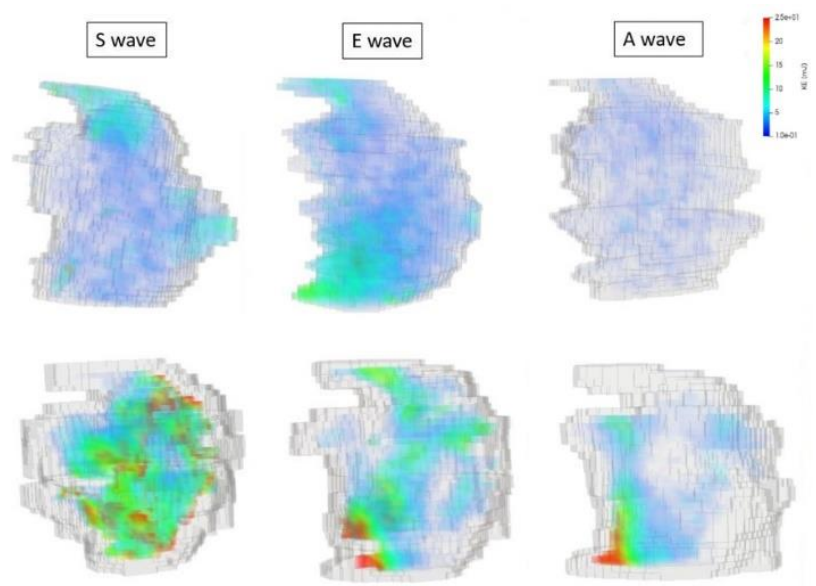

Figure 5. Analysis of the KE inside the LA during three different time frames for a healthy volunteer (first row) and a sick patient (second row).

\section{Conclusions}

In this study we developed a new approach for the analysis of a patient-specific model of atrial hemodynamics that is suitable for both healthy and sick patients. In particular, we designed a method that allows analyzing the blood flow inside the LA chamber and many other characteristics, which are linked to the velocity field. The developed method was based on the patient specific LA geometry, motion and velocities, thanks to the analysis of real clinical data. An initial evaluation of our framework was presented in order to study the effect of MVR on the inter-atrial blood flow, compared to healthy control group. In this initial testing, we observed that the analysis returned realistic blood flow patterns for both healthy volunteers and sick patients. In addition, the analysis seemed to confirm that patients affected by mitral regurgitation showed anatomical, morphological and hemodynamical changes that may reduce the washout of the LA chamber compared to the healthy group, which may lead to an increase of clot formation with a following increase of the risk of thromboembolism [12].

The comparison of the results obtained in MVR conditions against healthy left atria may provide a better understanding of hemodynamic blood flow patterns implications of mitral regurgitation.

\section{References}

[1] M. Enriquez-Sarano, C. W. Akins, Mitral Regurgitation, Review, Lancet April 7, 2009; 373: 1382-94

[2] F. Bursi, M. Enriquez-Sarano, Mitral Regurgitation After Myocardial Infraction: A Review, The American Journal of Medicine (2006) 119, 103-112.

[3] Raymond Y. Kwong, Cardiovascular Magnetic Resonance Imaging (cap.1,2,3), 2008.

[4] R.J. van der Geest, P. Garg, Advanced Analysis Techniques For Intra-cardiac Flow Evaluation From 4D Flow MRI, Current Radiology Report, May 20, 2016.

[5] A. T. Huber, N. Kachenoura, Cardiac MR Strain: A Noninvasive Biomarker of Fibrofatty Remodelling of the Left Atrial Myocardium, RSNA 2017.

[6] P. Getreuer, Chan-Vese Segmentation, Image Processing On Line 2012-08-08.

[7] M. Kanski, Per M. Arvisson, Left Ventricular Fluid Kinetic Energy Time Curves in Heart Failure From Cardiovascular Magnetic Resonance 4D Flow Data, Journal of Cardiovascular Magnetic Resonance, 2015, 17:111.

[8] G. G. Blume, C. J. Mcleod, Left Atrial Function: Physiology, Assessment, and Clinical Implications, European Journal of Echocardiography (2011), Vol. 12, pp. 421-430.

[9] E. Svalbring, A. Fredriksson, et al, Altered Diastolic Flow Patterns and Kinetic Energy in Subtle Left Ventricular Remodeling and Dysfunction Detected by 4D Flow MRI, PLOS ONE, August 17, 2016.

[10] M. Evin, N. Kachenoura, et al, Left Atrium MRI 4D-Flow in Atrial Fibrillation: Association with LA Function, Computing in Cardiology Conference, September 2015.

[11] M.Eliakim, J. Weinman , et al, Pulse Wave Velocity in Healthy Subjects and in Patients with Various Disease States, American Heart Journal, Vol. 82, Issue 4, October 1971, pp. 448-457.

[12] A. Masci, C. Corsi, et al, A Patient-specific Computational Fluid Dynamics Model of the Left Atrium in Atrial Fibrillation: Development and Initial Evaluation, Functional Imaging and Modelling of the Heart, 2017, pp. 392-400.

Address for correspondence.

Enrico Soldati

DEI, University of Bologna

Via Risorgimento 2, 40136 Bologna, Italy

E-mail address: cristiana.corsi3@ unibo.it 